\title{
Hashtags on Instagram: Self-created or Mediated by Best Practices and Tools?
}

\author{
Isabelle Dorsch \\ Heinrich Heine University Düsseldorf \\ isabelle.dorsch@hhu.de
}

\begin{abstract}
Social media enables conversations mediated through documents as texts, audio, images, or videos. Likewise, hashtags became an essential medium for social media communication. Instagram is well-known as one of the current platforms for hashtagging. This exploratory study investigates how hashtags used on Instagram became established in respect of selfcreation and best practices or tools. The analysis is based on data obtained from an online survey $(N=$ 1,006) of Instagram users. 55.7\% of the respondents use hashtags on Instagram. Only self-created hashtags are assigned by 41.4\%, whereas 58.6\% are (sometimes) inspired by others. Best practices and tools based on friends/other users or Instagram functions are more frequently used in contrast to offers from influencers or third-parties (e.g. guides, hashtag-sets). Furthermore, the majority does not intentionally use false hashtags. This study enables a first overview of the Instagram users' hashtagging creation behavior and selection process.
\end{abstract}

\section{Introduction}

Conversation on social media is mediated by documents (e.g. text, audio, image, and video) and content-describing user-generated tags. On Instagram, documents are especially images, videos, and ephemeral moments, while their content is described by hashtags like for example \#vacation if the posting is about vacations or shows vacation-related content. Therefore, it is not only important to know, for example, what kind of hashtags exist, when they are applied, or what motivations stand behind (hash)tagging, but also how they became established. Do Instagram users rather rely on self-created hashtags or do they apply best practices and tools for hashtagging? Little is known about the self-creation and creation by best practices and tools of hashtags, although such an analysis would add another important dimension to the overall hashtag and hashtagging analysis. Since hashtags serve as a further mean to communicate in social media it is important to follow this question. This exploratory study exactly intends to answer this question for hashtagging behavior in Instagram and sheds light on the hashtagging creation behavior and selection process on Instagram.

\subsection{Background: Hashtagging on Instagram}

Social media enables their users to communicate through a variety of channels using textual, audio, visual, or mixed media. Platforms as Twitter or Instagram also allow for using hashtags. A hashtag is composed of a \#-sign and a character string (e.g. \#happytobehear). "[By] using the \# character to mark particular keywords, [...] users communicate a desire to share particular keywords folksonomically" [14]. Its idea originates from a Twitter Tweet by Chris Messina in 2007 [18]. After this posting several platforms implemented a hashtag function and hashtagging on social media established [14]; still having its roots in the area of social tagging and user-generated knowledge representation $[25,26,36]$.

Nowadays, hashtags are integrated in our online and offline environment. As parts of online conversations or content captions, they play a role in understanding the interplay between media producers and media audiences. In such a computer-mediated discourse, hashtags indicate a shift "from online conversation to "searchable talk" " [7, 31, 38]. Moreover, they are also "an instrument for creative self-expression and language play" [15:51], started as a "rather peripheral typographic resource" and evolving "to an emblem of social media linguistic practice" [15:52].

The application Instagram is well-known for posting visual media, like for example picture postings, videos or so-called ephemeral moments known as "stories." The app's monthly active users numbered more than 1 billion as of June 2019 [16].

Research incorporating the analysis of hashtags in Instagram is diverse. In more general studies, hashtags serve as a filter to receive material for content analysis. For example, in order to receive access to political 
debates of Clinton/Trump supporter during the 2016 US presidential election, Schmidbauer et al. [30] analyzed Instagram postings collected through 16 hashtags related to the topic.

Several studies have investigated motivational factors for using hashtags on Instagram based on the Uses and Gratifications (U\&G) theory. The six motivations self-presentation, chronicling, inventiveness, information seeking, venting, and etiquette were identified by Erz et al. [9].

Sheldon et al. [32] compared American (individualist culture) and Croatian (collectivistic culture) undergraduate students' motivations of Instagram use. They point out self-promotion, social interaction, diversion, documenting, and creativity as gratifications influencing Instagram use for both cultures. According to the usage of hashtags, the American students use them mainly for documentation purpose. Similarly, the Croatian students do, however they further use them for other reasons like selfpromotion, social interaction, and creativity.

Motivational factors for hashtagging on Instagram, but also on other social media services as Twitter, Facebook, and LinkedIn, were the object of analysis in another study (composed of six empirical sub-studies), too. Here, the authors identified ten different motivations for the use of hashtags, namely amusing, organizing (especially on Twitter and Instagram), designing, conforming, trendgaging, bonding, inspiring, reaching, summarizing (especially on Instagram), and endorsing [28].

Besides studies investigating motivational factors, further hashtagging behavior is also of interest. An analysis of social tagging dynamics in respect of tag adoption patterns and the variety of tagging usage on Instagram reveals that the examined media (based on a dataset containing 9 million hashtags) amounts in a power law distribution, with the majority of media tagged with a few tags [10].

The descriptive power of hashtags was observed in a further study. Based on 1,000 Instagram pictures, certain hashtags, assigned by the picture posting owners were compared with hashtags study participants should choose from a list to describe the picture. For $66 \%$ of the chosen hashtags study participants would choose the same hashtags as the picture owners did in order to describe the content of the picture [12].

A hashtag content analysis of 14,649 hashtags from 1,000 Instagram images (divided into ten picture categories) shows, with $60,2 \%$ the majority of analyzed hashtags refers to content-related aspects of the posting, visible in the pictures. In contrast, hashtags communicating emotions or being full sentences are less often assigned [8]. Based on this a follow-up study on gender-specific hashtagging came to similar overall results and further found out that females tend to use more emotional hashtags, whereas males assign hashtags related to non-topical features, like technical aspects of the picture such as the camera type or Instagram related hashtags (e.g. \#IGers, \#instagood) [27].

Focusing on gender hashtagging differences of Malaysian food-related postings, the analysis of 1,382 Instagram images tagged with the hashtag \#Malaysianfood reveals women tend to use emotional and positive hashtags in comparison to male users [37].

The following studies consider more specific aspects of hashtagging. The hashtag \#like4like, belongs to the group of performativeness hashtags which means those hashtags are calling for an action [8, 26]. Similarly, \#goout or \#followmearound, \#like4like not only calls for the action to like a user's posting(s), but also promises to give (a) back-like(s) to those who liked the posting(s) [26, 39]. According to the hashtagging behavior on Instagram, it has been found that \#like4like does not really provoke more likes (after 2013) and that, contradictory to what the hashtag mediates, most users do not like back [39].

An analysis of 18,366 Instagram picture postings tagged with \#nofilter unveils that other as indicated $12 \%$ indeed used a filter for their image [29]. Thus hashtag can mediate false information, too.

Using participatory hashtag practices [22] some people promote their followers to upload images which match a suggested hashtag. A well-known example is Instagram's Weekend Hashtag Project (\#WHP).

There exist a variety of health- and disease-related hashtags on Instagram. For example, obvious hashtags addressing a certain disease (e.g. \#endometriosis) or aspects such as symptoms/sequelae of a disease (e.g. \#selfharm). When searching after \#selfharm, Instagram currently pops up a "Can we help?" message, explaining that posts with this hashtag "often encourage behavior that can cause harm and even lead to death" and the option to show posts, cancel the request or receive help [17]. Looking back, there were also times when - at some point - such hashtags were totally banned for the users. Consequently, variations (as for this case \#selfharmm, \#selfharmmm) emerged [21]. As a further result, a sub-group of secret hashtags established, especially used by adolescents [19, 21]. They are ambiguous or encrypted, so that they are less often or less quickly blocked. Those hashtags function as an entry to find (mental) health communities or conversations and offers the opportunity of being part of them. Apart from that, their users have to fear to be banned by Instagram to a lesser extent [5]. Those restrictions are not limited to health-related hashtags, but rather concerns a broader field of Instagram hashtags [5]. 
The previously stated cases have underlined in which ways hashtagging on Instagram is part of mediated online conversation. Likewise, they show which effects they can have and which consequences or restrictions might arise.

\subsection{Best Practices and Tools}

According to the latest definition of the Oxford dictionary [23] best practices are "[c]ommercial or professional procedures that are accepted or prescribed as being correct or most effective." Similarly, it is defined by the Cambridge dictionary [4] as "a working method or set of working methods that is officially accepted as being the best to use in a particular business or industry." Consequently, best practices can be seen as the result of a comparative process of an action with any alternative courses, whereby the action is linked to some outcome or goal [2]. Following these definitions and considering its origin from the field of economics, the application of the concept expanded over time. Nowadays, best practices are adapted in a variety of areas.

With the rise of social media, best practices incorporating or even solely for this scope evolved. For instance, a short Google search reveals that there are plenty of best practice guides and advice for the usage of social networking services like Facebook or Instagram. Staying with Instagram, they exist for special aspects or functions of the app as well. Entire guides and tools exclusively address how to hashtag on Instagram. From stating more universal advice, over specific user or company centered best practices to providing "trending hashtags" or hashtag sets (focusing on a specific purpose or topic), the offer is manifold diverse. The information can be embedded in news or blog postings, but there also exist YouTube videos, entire websites or applications (also listing or generating hashtag-sets and hashtag statistics), all dedicated to hashtagging best practices and tools for this purpose.

\begin{tabular}{c}
\hline DOGS \\
\hline \#tagstagram \#dog \#dogs \#dogsofinstagram \\
\#dogoftheday \#dogstagram \\
\#dogsofinstaworld \#dogs_of_instagram \\
\#doglover \#doge \#doggy \#doglovers \\
\#dogs_of_world \#doggie \#doggo \\
\#dogsofinsta \#instadog \#dogsandpals \\
\#mansbestfriend \#doggie \\
\hline
\end{tabular}

Table 1. Pre-defined hashtag set for Instagram postings addressing dog-related topics by the app Tagstagram
For example, Table 1 shows a pre-defined hashtag set for dog content postings provided by the iOS app Tagstagram. Within the app, users have the function "Copy Tags" to copy a whole hashtag set and to mark sets as "Favorite" [3]. Equivalent to this is the android app Tagify [6] or one of the many other applications or websites which come across with such a feature. One of the first hashtagging set providers might be TagsForLikes, but with the latest update in 2013 it is no longer up-to-date [35]. Nevertheless, according to its founder, TagsForLikes were used by over 5 million users in more than 129 million pictures [20].

As a second example and having a closer look on some up-to-date guides (e.g. $[1,13])$, they often provide some general reasons for using hashtags on Instagram, state what changed and what is currently important to know (like with these examples such guides often refer to a specific year for which they are "valid"), state concrete "tips and tricks" like "[m] ake sure that hashtag means what you think it means," [1] or to better avoid popular Instagram hashtags like \#like4like, \#followme, or \#tagsforlikes since they attract the wrong audience ("bots, spammers, and others users who have no intention of engaging with you in any meaningful way" [1]). Thereby, often is their main focus to increase the engagement on Instagram.

In our study, the term "Best Practices" means all suggested and well-established practices; "Tools" summarizes all available instruments for hashtagging. We distinguish between the following kinds of best practices and tools:

- Pre-defined hashtag-sets,

- Hashtag statistics,

- Hashtag guidelines,

- Direct hashtags recommendations by friends,

- Direct hashtag recommendations by influencers,

- Other users' or friends' hashtags seen on Instagram,

- Autocompletion on Instagram,

- Number of hashtags on Instagram,

- Instagram hashtag search function, and

- User's own practices, criteria, and experiences.

\subsection{Best Hashtagging Practices and Tools on Instagram}

Hashtags are essential parts of social media communication. As the literature review shows, there exist studies about hashtags on Instagram in general and specifically on certain hashtagging behaviors and motivations, but there is still need to examine how hashtags on Instagram are created by their users. 
In order to get to know more about the general role of hashtagging in computer-mediated conversation it is also important to analyze how hashtags are inspired by other influential factors as best hashtagging practices and tools and to what extent users rely on their own when using hashtags. Therefore, this exploratory study concentrates on answering the following research questions:

RQ1: How many Instagrammers use hashtags and for what?

RQ2: How are best hashtagging practices and tools used on Instagram and to what extent do users create hashtags on their own?

RQ3: Do Instagram users intentionally assign false hashtags?

We focus on Instagram as platform to be analyzed since it is one of the current platforms which is, similar to Twitter, strongly used for hashtagging in social media [24] and nowadays "the most prominent hashtagging platform" [31:7]. Furthermore, the possibility to annotate up to 30 hashtags as well as the establishment of new hashtags make the application especially interesting for this analysis. For example, hashtag phenomena as \#like4like or even an entire platformspecific hashtag category called "Insta"-Tags (e.g. \#IGers; \#insta + any kind of term, like \#instaartists) established on Instagram [8, 39].

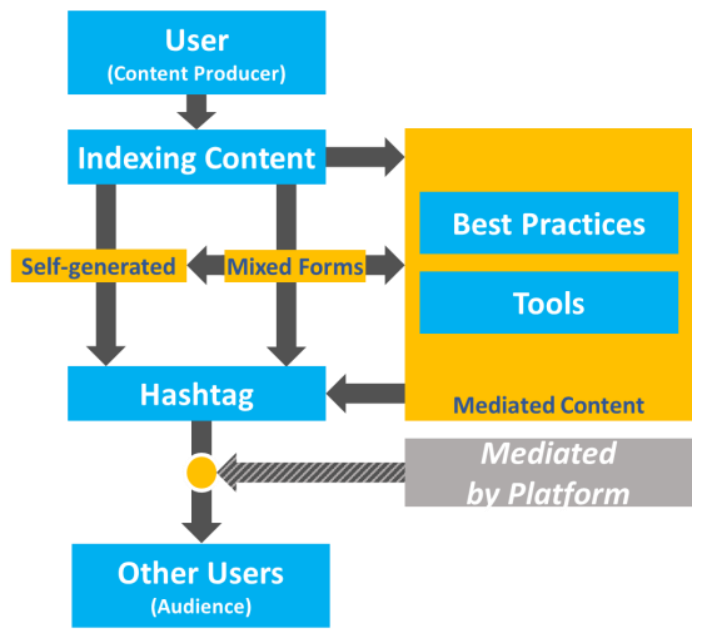

Figure 1. Research model

Based on our research questions and the literature background the following research model (Figure 1) underlays our study. Referring to Instagram, a user has the possibility to index content to be published with hashtags (e.g. Instagram pictures, videos, etc.) or otherwise just to add a caption or post the content without any further textual information. If adding hashtags to the posting, they can be self-generated, based on mediated content, or based on mixed forms of both prior stated variations. The hashtagged content will then be published on Instagram and is available for its audience - other Instagram users. Thereby, the process of hashtagging contributions normally happens during the publishing process, however, for some content like pictures or videos, it is also possible that users add their hashtags past publishing in the comment section or modify, add, or delete them from their caption. Some hashtags are also mediated by the platform needing a confirmation to be displayed with their indexed content or being totally blocked for the users.

\section{Methods}

In order to answer the research questions for this exploratory study, a questionnaire for an online survey was developed. This approach was chosen to receive first general insights about this topic. Since the information questioned cannot be obtained directly from Instagram users' profiles, a survey provides a means of asking users about their preferences and behaviors. Although, experiments, field research, or interviews allow for gathering data on such user behavior, they were not suitable for this study. Experiments have the advantage to be able to exactly note down every behavioral aspect, but in the same way, the setting would have caused a man-made labor situation, not referring to the reality. Directly observing users within a field research context would involve certain problems. For example, people probably might not allow to observe them in such a context. Besides, both of these approaches would rather illustrate a current situation for specific cases as reveal more general statements. Interviews employ a solid starting point for exploratory studies. However, based on the existing literature and study structure, we choose an online survey setting. Furthermore, this approach allows us to reach a wide audience and to generate enough empirical data for the analysis.

\subsection{Survey}

Overall, the questionnaire is composed of 26 items (see Appendix). However, as can be obtained, each of these items does not apply to every survey participant. The first questions and notes (3.-9.) refer to a user's general Instagram usage and account settings. Questions 10. and 11. ask for the general usage and importance of hashtags. If participants stated to use hashtags the next question 12. concerns the area of application, followed 
by asking if one uses only self-created hashtags or if they are (sometimes) inspired by others (13.). Depending on the answer further questions concentrate on the application (14.-18.) or non-application (19.-22.) of Instagram hashtagging best practices or tools. The questionnaire concludes with items 23. to 26., surveying demographic statements and providing space for final comments.

The survey initially was composed in English language, followed by a German translation. Overall, it is based on our research model, the knowledge of the current state of research as well as the best practices and tools.

In total 7 persons $(2=$ male; $5=$ female $)$ pre-tested the survey and the received feedback where considered for or integrated in the survey. Umfrageonline ${ }^{1}$ was chosen to host the survey.

\subsection{Data Collection, Cleansing, \& Analysis}

The distribution of the survey took place during May 12, until June 5, 2019. According to a nonprobability sampling, we digitally and physically promoted the survey participation. Therefore, the survey was spread via social media (Instagram, Reddit, Facebook, and Twitter), online forums, e-mail listservs, via flyers in Germany (mainly at places in Düsseldorf and Leverkusen), and Switzerland (Buchs and Chur).

During the data collection, we received the feedback that the gender denotation "divers" in the English questionnaire should be better changed into "other." This was done on May 22, 2019.

It was necessary to clean up the data gathered from our survey. Form the overall 1,338 participants, 1,156 completed the questionnaire. Since in question 3. 145 respondents selected not to use Instagram, 1,011 remain. However, five of them could still not be considered for the analysis. Two indicated in question 4. to have " 0 " Instagram account(s). Two further participants were removed as well because they selected one and two for their age and one due to problems with the software of Umfrageonline. Therefore, the data cleansing results in a total of 1,006 participants, considered for the analysis.

Due to the inclusion of filter questions (see Appendix), the overall number of respondents varies for some questions. For a better understanding Tables 2-5 therefore include an "overall N" indicating how many persons answered this question overall. These also incorporate respondents which selected the statement "No experience" or "I don't know" which is not further analyzed within these tables.

\footnotetext{
${ }^{1}$ https://www.umfrageonline.com
}

\section{Results}

In our study $(\mathrm{N}=1,006), 63.1 \%$ of Instagram users identify as female, $33.2 \%$ as male, and $2.8 \%$ as other. The remaining $0.9 \%$ preferred to make no statement about their gender. Although the worldwide gender distribution on Instagram is more balanced (52\% female and $48 \%$ male) [34], women generally are more likely to fill out online surveys [33].

Based on the age groups identified by Fietkiewicz [11] 52\% of our participants belong to Generation Y (born between 1980 and 1996), 42.7\% to Generation Z (born after 1996), 5\% to Generation X (born between 1960 and 1980), and $3 \%$ to the so-called "Silver Surfers" (born before 1960).

From a total of 55 countries, the majority come from Germany (37.7\%) and the United States (35.1\%), followed by Canada (5.8\%), the United Kingdom $(5.3 \%)$, Australia (2.3\%), and the Netherlands (1.3\%).

With $64.8 \%$ nearly two-thirds report to have one Instagram account, whereas $23.4 \%$ have two accounts and $11.7 \%$ three or up to seven accounts. However, one person indicated to have 18 accounts.

Considering the visibility settings for Instagram accounts the distribution is nearly fifty-fifty. This means $50.1 \%$ have their profile open to the public and conversely, $49.9 \%$ set their profiles on private mode, only allowing confirmed users to see their postings.

Having a closer look on the follower numbers, $61.8 \%$ respondents have between 100 and 999 Instagram follower, followed by $31.0 \%$ respondents with between 1 and 99; 5.2\% respondents with between 1,000 and 9,999; $0,7 \%$ respondents with between 10,000 and 99,999 respondents; and $0.3 \%$ respondents with between 100,000 and 499,999 followers. To have 0 Instagram follower was selected by $1 \%$.

Nearly all use their account only by themselves $(98.4 \%)$ and just a very small amount shares it with further persons $(1.6 \%)$.

\section{RQ1: How many Instagrammers use hashtags and for what?}

When analyzing the usage of hashtagging best practices and tools, it is also important to look at how many Instagram users make use of hashtags. More than half $(55.7 \%)$ of the 1,006 respondents use hashtags on Instagram.

Table 2 shows how important hashtags for searching postings as well as for using them in own contributions posted on Instagram are. $20.7 \%$ indicated they are rather important for searching, whereas $28.6 \%$ find them very 
Table 2. Hashtag importance for searching contributions by other Instagram users and for the usage within own contributions

\begin{tabular}{c|c|c|c|c|c|c|c|c|c}
\hline 7 & 6 & 5 & 4 & 3 & 2 & 1 & Median & IQR & N \\
\hline \multicolumn{8}{|c|}{ Searching contributions by others (e.g. pictures, videos, etc.) } \\
\hline $11.5 \%$ & $16.0 \%$ & $20.7 \%$ & $13.4 \%$ & $11.4 \%$ & $10.9 \%$ & $16.3 \%$ & 4 & 4 & 958 \\
\hline \multicolumn{8}{|c|}{ Usage within your own contributions (e.g. pictures, videos, etc.) } \\
\hline $10.2 \%$ & $11.2 \%$ & $11.5 \%$ & $13.8 \%$ & $11.8 \%$ & $13.0 \%$ & $28.6 \%$ & 3 & 4 & 934 \\
\hline 7 = Very important, 6 = Important, 5 = Rather important, 4= Neutral, 3 = Rather unimportant, 2 = Unimportant, 1 = Very unimportant \\
\hline \multicolumn{8}{c}{ Overall $N=1,006$} \\
\hline
\end{tabular}

Table 3. Frequency of hashtag usage for Instagram activities

\begin{tabular}{|c|c|c|c|c|c|c|c|c|c|}
\hline 7 & 6 & 5 & 4 & 3 & 2 & 1 & Median & IQR & $\mathrm{N}$ \\
\hline \multicolumn{10}{|c|}{ For my picture postings } \\
\hline $41.1 \%$ & $23.3 \%$ & $22.5 \%$ & $4.8 \%$ & $6.6 \%$ & $0.9 \%$ & $0.7 \%$ & 6 & 2 & 559 \\
\hline \multicolumn{10}{|c|}{ For my video postings } \\
\hline $30.5 \%$ & $15.2 \%$ & $18.4 \%$ & $8.6 \%$ & $10.9 \%$ & $1.4 \%$ & $14.9 \%$ & 5 & 4 & 348 \\
\hline \multicolumn{10}{|c|}{ For my story postings } \\
\hline $6.7 \%$ & $6.7 \%$ & $26.0 \%$ & $15.9 \%$ & $17.1 \%$ & $2.6 \%$ & $25.0 \%$ & 4 & 3,75 & 416 \\
\hline \multicolumn{10}{|c|}{ For my profile description } \\
\hline $1.4 \%$ & $3.3 \%$ & $6.7 \%$ & $6.1 \%$ & $5.5 \%$ & $4.1 \%$ & $72.9 \%$ & 1 & 1 & 491 \\
\hline \multicolumn{10}{|c|}{ In comments to my own postings } \\
\hline $1.8 \%$ & $4.5 \%$ & $10.9 \%$ & $12.1 \%$ & $17.6 \%$ & $1.4 \%$ & $51.6 \%$ & 1 & 3 & 494 \\
\hline \multicolumn{10}{|c|}{ In comments to other postings } \\
\hline $1.4 \%$ & $2.6 \%$ & $17.2 \%$ & $12.0 \%$ & $18.1 \%$ & $2.0 \%$ & $46.7 \%$ & 3 & 3 & 507 \\
\hline \multicolumn{10}{|c|}{$7=$ Very frequently, $6=$ Frequently, $5=$ Occasionally, $4=$ Rarely, $3=$ Very rarely, $2=\mathrm{I}$ only tried it once, $1=$ Never } \\
\hline
\end{tabular}

unimportant for their own contributions. Although, it becomes apparent that the opinions differ and have a width range, also demonstrated by the interquartile range (IQR) of 4 and the medians. The inclusion of all participants for this question might be a reason for such a broad distribution.

There is a clear trend for the frequency of hashtag usage for different activities on Instagram (Table 3). Especially own picture and video postings are (very) frequently hashtagged. In contrast, the clear majority of $72.9 \%$ never used hashtags for their profile description. Likewise, is this for hashtags in comments. Here, $51.6 \%$ never use them in own comments to their posting and with $46.7 \%$ this applies for comments to other postings.

RQ2: How are best hashtagging practices and tools used on Instagram and to what extent do users create hashtags on their own?

Considering all participants using hashtags $(\mathrm{N}=$ $560), 41.4 \%$ of them only use self-created hashtags. The remaining $58.6 \%$, and thus the majority, reported to create their hashtags (sometimes) inspired by others and not solely by their own. Following this, participants using inspired hashtags were asked how important certain hashtagging best practices and tools are for them and how frequently they use them. The frequency of applying hashtagging best practices or tools can be obtained from Table 4. Overall, these results are quite similar to the ones found for the importance. Therefore, we focus in more detail only on one of these two aspects, namely the frequency.

For the usage of pre-defined hashtag-sets (47.9\%), hashtag statistics from third-parties $(65.2 \%)$, overall hashtag guidelines $(54.5 \%)$, and direct hashtag recommendations or practices received from influencers (48.4\%) the proportional majority stated to never use those. Direct hashtag recommendations or practices by friends $(26.3 \%)$, users'/friends' hashtags directly seen on Instagram (30.3\%), Instagram's hashtag autocompletion function $(24.7 \%)$, the information about the number of times a hashtag is assigned on Instagram $(23.4 \%)$, and the Instagram hashtag search function $(24.1 \%)$ are mostly used occasionally (again based on the proportional majority). Especially for the last stated 4 aspects, Instagram hashtag functions and users'/friends' hashtags, the usage can be considered as rather frequent since many also stated to use it 


\section{Discussion}

Our exploratory study investigated the application of hashtagging best practices and tools on Instagram in contrast to the self-creation of hashtags. In doing so, it shows how far best practices and tools influence the establishment of hashtags. For the study, we conducted an online survey with 1,006 participants.

In respect to $\mathbf{R Q 1}$, more than half of the respondents $(55.7 \%)$ use hashtags on Instagram. The importance of searching after contributions with hashtags or using them in own contributions on Instagram generally varies but is slightly more important for searching with hashtags. Considering the frequency of application, hashtags are predominantly used in the picture and video postings, whereas profile descriptions, and comments to own or other postings are generally not or rarely assigned with hashtags.

The results for RQ2 show that $41.4 \%$ of Instagram users use self-created hashtags only, whereas $58.6 \%$ are (sometimes) inspired by others. Interestingly, best practices or tools connected to an Instagram user's friend (or other users), and functions offered by Instagram itself are rather frequently used in contrast to Influencer or third-party offers.

Using intentionally false hashtags as questioned in RQ3 is something the most have never done. This applies similarly to users (sometimes) inspired by best practices and tools as for those only using their own hashtags.

The overall results have shown that the hashtagging creation behavior and selection process is not only solely based on self-created hashtags or certain bestpractices and that best practices appealing more personal or directly referring to Instagram are more frequently used. Instagram users might rely on more personal and individual inspiration in order to give their hashtags a more personal expression. One participant also gave us the feedback that his or her hashtags often serve as a medium to communicate jokes or to add ironic or humoristic elements to the posting (e.g. \#hashtag) and that this is more important to him or her instead of making the contributions searchable. For the participant, such language plays function much better as hashtag than jokes within the normal text caption [personal communication]. Likewise, some other respondents stated to use them to make fun or jokes.

The data of this study is limited to a non-probability sample. It is not possible to create a sample based on the total Instagram population since such data is not freely accessible. However, this problem is - unfortunately well-known in social media research and not limited to this study.

This study generated first insights about Instagram users' hashtag self-creation and the creation mediated by best practices and tools; it helps to analyze hashtags as part of social media communication. Our questionnaire also contained a few basic open questions. The conduction of further in-depth interviews could provide more detailed structured information for a better understanding of these first findings and broaden the study scope. For example, users could be asked about their motivations to apply or not apply best practices and tools and if they do so, looking back to their behavior, achieve to satisfy their motivations. Interviews also enable the possibility to exactly address the self-creation behavior or specific best practices and tools suggested by best practice guides, recommendations, etc. addressed in this study. Besides, a closer examination could identify if there exist gender-, age- or countryspecific differences within the application and in how far account aspects like the number of followers, profile visibility or number of account administrators influence best hashtagging practices and tools.

Focusing on the platform, it is important as well to analysis in how far the hashtag mediation by Instagram affects the online communication through hashtags. Furthermore, there is also need to analyze how hashtags are perceived by their audience in more detail. For example, the best practice to hide hashtags in the comment section or caption of picture and video contributions. How is this perceived by the users and what does it communicate to them?

\section{References}

[1] Aynsley, M., "The 2019 Instagram Hashtag Guide-How to Use Them and Get Results", 2019.

https://blog.hootsuite.com/instagram-hashtags/

[2] Bretschneider, S., F.J. Marc-Aurele, and J. Wu, “"Best practices' research: A methodological guide for the perplexed", Journal of Public Administration Research and Theory 15(2), 2005, pp. 307-323.

[3] BYOApps LLC, "Tagstagram - Hashtag Generator", 2019. https://apps.apple.com/us/app/tagstagram-hashtaggenerator/id587756737

[4] Cambridge University Press, "best practice", 2019. https://dictionary.cambridge.org/dictionary/english/bestpractice

[5] Dapper Goat Social Media, "BANNED INSTAGRAM

HASHTAGS 2019”, 2019.

https://www.dappergoat.com/blog/banned-instagramhashtags-2019/

[6] Dev, G., "Tagify - Best Hashtags for Instagram", 2019. https://play.google.com/store/apps/details?id=com.gongadev. hashtagram\&hl=de

[7] Dickinson, P., “'B/w U \& me': The Functions of Formulaic Language in Interactional Discourse on Twitter", The Linguistics Journal 7(1), 2013, pp. 7-38.

[8] Dorsch, I., "Content description on a mobile image sharing service: Hashtags on Instagram", Journal of Information Science Theory and Practice 6(2), 2018, pp. 46- 
61.

[9] Erz, A., B. Marder, and E. Osadchaya, "Hashtags: Motivational drivers, their use, and differences between influencers and followers", Computers in Human Behavior 89(July), 2018, pp. 48-60.

[10] Ferrara, E., R. Interdonato, and A. Tagarelli, "Online popularity and topical interests through the lens of Instagram", The Proceedings of the 25th ACM Conference on Hypertext and Social Media, ACM (2014), 24-34.

[11] Fietkiewicz, K., "Jumping the digital divide: How do 'silver surfers' and 'digital immigrants' use social media?", Networking Knowledge 10(1), 2017, pp. 5-26.

[12] Giannoulakis, S., and N. Tsapatsoulis, "Evaluating the descriptive power of Instagram hashtags", Journal of Innovation in Digital Ecosystems 3(2), 2016, pp. 114-129.

[13] Gilbert, S., "New Rules for Instagram Hashtags: How to Find the Right Hashtags for Your Business", 2019. https://later.com/blog/instagram-hashtags/ [14] Halavais, A., "Structure of Twitter: Social and technical", In K. Weller, A. Bruns, J. Burgess, M. Mahrt and C. Puschmann, eds., Twitter and Society. Peter Lang, New York, NY, 2014, 29-41.

[15] Heyd, T., and C. Puschmann, "Hashtagging and functional shift: Adaptation and appropriation of the \#", Journal of Pragmatics 116, 2017, pp. 51-63.

[16] Instagram, "Instagram Statistics", 2019.

https://instagram-press.com/our-story/

[17] Instagram, "Can we help?", 2019.

https://www.instagram.com/explore/tags/selfharm/?hl=en

[18] Messina, C., "How do you feel about using \# (pound)

for groups. As in \#barcamp [msg]? @ chrismessina

(Twitter)", 2007.

https://twitter.com/chrismessina/status/223115412

[19] Metcalfe, H., A. Beesley, C. Watson, et al.,

"\#SpeakingTheirLanguage: Engaging with young people's mental health in a digital landscape", Clinical Psychology

Forum(307), 2018, pp. 9-14.

[20] Mohiuddin, Z., "Products", https://www.zaheer.me/

[21] Moreno, M.A., A. Ton, E. Selkie, and Y. Evans, "Secret Society 123: Understanding the language of self-harm on Instagram", Journal of Adolescent Health 58(1), 2016, pp. 78-84.

[22] Oh, C., T. Lee, Y. Kim, S. Park, and B. Suh,

"Understanding Participatory Hashtag Practices on

Instagram: A Case Study of Weekend Hashtag Project",

CHI'16 Extended Abstracts, ACM (2016), 1280-1287.

[23] Oxford University Press, "best practice", 2019.

https://en.oxforddictionaries.com/definition/best_practice

[24] Pennington, D.R., and L. Spiteri, eds., Social Tagging in a Linked Data Environment, Facet Publishing, London, UK, 2019.

[25] Peters, I., Folksonomies. Indexing and retrieval in web 2.0, De Gruyter Saur, Berlin, 2009.

[26] Peters, I., and W.G. Stock, "Folksonomy and information retrieval", Proceedings of the American Society for Information Science and Technology 44(1), 2007, pp. 128.

[27] Philipps, J., and I. Dorsch, "Gender-specific tagging of images on Instagram”, Social Computing and Social Media. Design, Human Behavior and Analytics. HCII 2019. Lecture Notes in Computer Science, Vol .11578., Springer (2019),
396-413.

[28] Rauschnabel, P.A., P. Sheldon, and E. Herzfeldt, "What motivates users to hashtag on social media?", Psychology and Marketing 36(5), 2019, pp. 473-488.

[29] Santarossa, S., P. Coyne, and S.J. Woodruff, "Exploring \#nofilter images when a filter has been used", International Journal of Virtual Communities and Social Networking 9, 2017, pp. 54-63.

[30] Schmidbauer, H., A. Rösch, and F. Stieler, "The 2016 US presidential election and media on Instagram: who was in the lead?", Computers in Human Behavior 81, 2018, pp. 148-160.

[31] Sheldon, P., E. Herzfeldt, and P.A. Rauschnabel, "Culture and social media: the relationship between cultural values and hashtagging styles", Behaviour and Information Technology(in press), 2019, pp. 1-13.

[32] Sheldon, P., P.A. Rauschnabel, M.G. Antony, and S. Car, "A cross-cultural comparison of Croatian and American social network sites: Exploring cultural differences in motives for Instagram use", Computers in Human Behavior 75, 2017, pp. 643-651.

[33] Smith, W.G., "Does gender influence online survey participation? A record-linkage analysis of university faculty online survey response behavior", Eric Ed501717, 2008.

[34] Statista, "Distribution of Instagram users worldwide as of April 2019, by gender", 2019.

https://www.statista.com/statistics/802776/distribution-ofusers-on-instagram-worldwide-gender/

[35] TagsForLikes, “\#TagsForLikes”, 2013.

https://www.tagsforlikes.com/

[36] Vander Wal, T., "No Title", 2007.

http://www.vanderwal.net/folksonomy.html

[37] Ye, Z., N.H. Hashim, F. Baghirov, and J. Murphy,

"Gender Differences in Instagram Hashtag Use", Journal of Hospitality Marketing and Management 27(4), 2018, pp. 386-404.

[38] Zappavigna, M., "Ambient affiliation: A linguistic perspective on Twitter", New Media and Society 13(5), 2011, pp. 788-806.

[39] Zhang, Y., M. Ni, W. Han, and J. Pang, "Does \#like4like indeed provoke more likes?", Proceedings of the International Conference on Web Intelligence (WI'17), (2017), 179-186. 


\section{Appendix}

\begin{tabular}{|c|c|c|c|}
\hline \# & Question/Note & Answer items & Likert \\
\hline 1. & Please choose a language. & English/German & \\
\hline 2. & Cover and survey note & $\begin{array}{l}\text { Includes the following statements: Welcoming, topic, purpose, required time, } \\
\text { data analysis and privacy, expression of thanks, contact information }\end{array}$ & \\
\hline 3. & Do you use Instagram? ${ }^{*}$ & yes/no [direction to the end page] & \\
\hline 4. & How many Instagram accounts do you have? * & $\begin{array}{l}\text { Dropdown menu: } 0-20,20+ \\
{[0=\text { direction to the end page] }}\end{array}$ & \\
\hline 5 . & $\begin{array}{l}\text { Intermediate text page } \\
\text { [Appears only if selected "more than one account" } \\
\text { in question } 4 . \text { ] }\end{array}$ & $\begin{array}{l}\text { "Please note, since you have more than one Instagram account, please focus } \\
\text { on all further questions on the Instagram account you post content the most." }\end{array}$ & \\
\hline 6. & $\begin{array}{l}\text { Is your Instagram account open to the public or } \\
\text { private? } *\end{array}$ & open to the public/private (a request is necessary) & \\
\hline 7. & $\begin{array}{l}\text { How many follower does your Instagram account } \\
\text { have? } *\end{array}$ & $\begin{array}{l}\text { Dropdown menu: 0/1-99/100-999/1,000-9,999/10,000-99,999/100,000- } \\
499,999 / 500,000-999,999 />=1,000,000\end{array}$ & \\
\hline 8. & $\begin{array}{l}\text { Please indicate, what applies to your Instagram } \\
\text { account: } *\end{array}$ & $\begin{array}{l}\text { I'm the only person using this account/It is a shared account with additional } \\
\text { persons using this account }\end{array}$ & \\
\hline 9. & $\begin{array}{l}\text { Intermediate text page } \\
\text { [Appears only if selected "shared account" in } \\
\text { question 8.] }\end{array}$ & $\begin{array}{l}\text { "Please note, since you are sharing the Instagram account, please focus on all } \\
\text { further questions on your own (inter)actions and behavior with this shared } \\
\text { Instagram account." }\end{array}$ & \\
\hline 10. & $\begin{array}{l}\text { Do you use hashtags on Instagram? }{ }^{*} \\
\text { Note: Hashtags are a composition of \# and a } \\
\text { character string, for example \#summer }\end{array}$ & yes/no [direction to question 23.] & \\
\hline 11. & How important are hashtags on Instagram to you? $*$ & $\begin{array}{l}\text { for searching contributions by others (e.g. pictures, videos, etc.) } \mid \text { for the usage } \\
\text { within your own contributions (e.g. pictures, videos, etc.) }\end{array}$ & $\mathrm{x}$ \\
\hline 12. & $\begin{array}{l}\text { For which of your activities on Instagram do you } \\
\text { use hashtags and how frequently? * }\end{array}$ & $\begin{array}{l}\text { for my picture postings } \mid \text { for my video postings | for my story postings | for my } \\
\text { profile description | in comments to my own postings | in comments to other } \\
\text { postings }\end{array}$ & $\mathrm{x}$ \\
\hline 13. & How do you create your hashtags? $*$ & $\begin{array}{l}\text { they are (sometimes) inspired by others (e.g. friends, recommendations, guides, } \\
\text { etc.)/they are only self-created [direction to question 19.] }\end{array}$ & \\
\hline 14. & $\begin{array}{l}\text { How important are best practices or tools for your } \\
\text { own hashtagging behavior on Instagram? } *\end{array}$ & $\begin{array}{l}\text { usage of pre-defined hashtag-sets (from web pages or apps) | hashtag statistics } \\
\text { (from third-parties) | overall hashtag guidelines (provided on blogs, websites, } \\
\text { Youtube, etc.) | direct hashtag recommendations or practices by friends | direct } \\
\text { hashtag recommendations or practices by influencers | based on users'/friends' } \\
\text { hashtags directly seen on Instagram | autocompletion hashtag recommendation } \\
\text { on Instagram | number of times a hashtag is assigned on Instagram | Instagram } \\
\text { hashtag search function | based on my own practices, criteria and experiences }\end{array}$ & $\mathrm{x}$ \\
\hline 15. & $\begin{array}{l}\text { How frequently do you use the following best } \\
\text { practices or tools within your own hashtagging } \\
\text { behavior on Instagram? } *\end{array}$ & [same items as in 14.] & $\mathrm{x}$ \\
\hline 16. & $\begin{array}{l}\text { How frequently do you intentionally use false } \\
\text { hashtags on Instagram - for instance - to receive } \\
\text { more views, likes, followers, etc.? } *\end{array}$ & $\begin{array}{l}\text { very frequently/frequently/occasionally/rarely/very rarely/I only tried it } \\
\text { once/never/I don't know }\end{array}$ & \\
\hline 17. & $\begin{array}{l}\text { Which specific best hashtagging practices or tools } \\
\text { do you use? }\end{array}$ & Blank box & \\
\hline 18. & $\begin{array}{l}\text { Do you have a specific reason why you apply best } \\
\text { hashtagging practices or tools? }\end{array}$ & Blank box [direction to question 23.] & \\
\hline 19. & $\begin{array}{l}\text { How frequently do you intentionally use false } \\
\text { hashtags on Instagram - for instance - to receive } \\
\text { more views, likes, followers, etc.? }{ }^{*}\end{array}$ & $\begin{array}{l}\text { very frequently/frequently/occasionally/rarely/very rarely/I only tried it } \\
\text { once/never/I don't know }\end{array}$ & \\
\hline 20. & $\begin{array}{l}\text { Do you know that there exist best hashtagging } \\
\text { practices and tools (e.g. asking friends, } \\
\text { recommendations, guides, etc.)? } *\end{array}$ & yes/no [direction to question 23.] & \\
\hline 21. & $\begin{array}{l}\text { Did you ever inform yourself about such best } \\
\text { practices or tools? } *\end{array}$ & yes/no [direction to question 23.] & \\
\hline 22 . & $\begin{array}{l}\text { Do you have a specific reason why you don't apply } \\
\text { best hashtagging practices or tools? }\end{array}$ & Blank box & \\
\hline 23. & What is your age? $*$ & Dropdown menu: 1-99 & \\
\hline 24. & What is your gender? $*$ & female/male/other/prefer not to say & \\
\hline 25 . & Where are you currently located? $*$ & Dropdown menu: Country list & \\
\hline 26. & $\begin{array}{l}\text { Do you have any additional comment you want to } \\
\text { share with us? }\end{array}$ & Blank box & \\
\hline
\end{tabular}

Notes: \# in the same box were displayed at the same time; * required questions; comments in [] contain information about the query sequence 